\title{
Measurement of DC currents in the power grid by current transformer
}

\author{
Pavel Ripka ${ }^{1}$, Karel Draxler ${ }^{1}$, and Renata Styblikova ${ }^{2}$ \\ ${ }^{1}$ Czech Technical University, 16627 Prague, Czech Republic \\ ${ }^{2}$ Czech Metrologic Institute, Prague, Czech Republic
}

DC currents in power grids are mainly caused by geomagnetic activity especially during magnetic storms. It is desirable to monitor these currents to prevent saturation of transformers, which may cause blackout. However, adding DC current sensors to existing installations would be very costly. We suggest to convert some of the existing current transformers to fluxgate DC current sensors by injecting AC excitation current into their secondary winding. We successfully tested this concept on 500 A current transformer. The achievable accuracy is $10 \%$ for DC currents and $1.5 \%$ for AC currents, which is sufficient for protection and monitoring purposes. We analyze the DC current sensitivity dependence on the (changing) grid impedance, and we show that the sensitivity can be stabilized by controlling the secondary voltage component at the excitation frequency. Excitation current injected into the grid also depends on the grid impedance, but for realistic conditions it is below $2 \mathrm{~A}$.

Index Terms—current sensor, fluxgate sensor, current transformer, DC current measurement

\section{INTRODUCTION}

$\mathrm{G}^{\mathrm{s}}$ eomagnetic storms are mainly caused by anomalous Sun activity. During these storms the Earth's magnetic field changes by hundreds and thousands of nanoTeslas during several minutes. These abrupt field changes induce DC telluric currents and also currents in pipelines and power networks [1]. Largest geomagnetically induced DC currents in power grids in the order of hundreds Amps are measured at higher latitudes such in Canada or Scandinavia, where the geomagnetic activity is large and the conductor loops are also large due to small numbers of transformers in large areas. These currents may cause saturation of transformers resulting in tripping of protective relays and possible blackout. In Europe the typical geomagnetically induced currents are tens of Amps as the geomagnetic field is more stable and the loops are smaller. New power transformers are built to be more DC tolerant after the large geomagnetic blackout in Canada. However DC currents still may cause local problems including generation of higher harmonics and potentially cause grid instability. Prediction of magnetic storms and monitoring of DC currents in multiple points of the grid can prevent problems. Optical current sensors and currents sensors based on Hall technology are affected by DC offset drifts [2], while GMI (Giant Magnetoimpedance) and MR (Magnetoresistive) current sensors have limited dynamic range [3]. The off-theshelf fluxgate current sensors are bulky and not openable, so they require to disconnect the measured conductor in order to mount them [4]. It would be very expensive to mount these DC current sensors inside the existing $200-700 \mathrm{kV}$ substations. Therefore the industrial requirement is to use existing current transformers to monitor also DC currents. It is possible to use computational methods for the detection and correction of the saturated current transformer. These methods based on decaying DC components currents are computational extensive and they do not allow to estimate the DC current value [5].

Manuscript received July 11, 2012. Corresponding author: P. Ripka (email: ripka@fel.cvut.cz).

Digital Object Identifier inserted by IEEE
There are other sources of DC offset current in the power grid: transformer-less power converters both on the energy source and consumption side and half-bridge rectified appliances. The possibilities of measuring DC currents in these cases are discussed in [6]. The authors monitor DC voltage component instead of the current and thus rely on resistive part of the grid impedance. This method can be effective only for zero detection for compensation purposes, as the mentioned impedance is unknown and therefore calibration is not possible. Thus it cannot be used for our application.

In [7] we have shown that DC current component in the current measured by current transformer in the electrical energy meter can be detected by monitoring the $2^{\text {nd }}$ harmonic voltage in the secondary winding. Both AC and DC current components should be present in order to utilize this effect. However, in our case the requirement was to measure DC current even without any AC current component.

\section{CT FluXgate CURRENT SENSOR}

In our case the available transformer type CLA 2.2 had 500 $\mathrm{A} / 5 \mathrm{~A}$ current ratio and the rated output load of $5 \mathrm{VA}$, corresponding with $0.2 \Omega$ load resistor. Thus the maximum output voltage was $1 \mathrm{~V}$.

To be able to measure the primary $\mathrm{DC}$ current $\mathrm{I}_{1 \mathrm{DC}}$ even without AC component, we applied $370 \mathrm{~Hz}$ AC sinewave excitation into the secondary winding of the current transformer and created fluxgate current sensor [4]. Compared to the traditional current sensors of this type such as [8] we did not used separate excitation and sensing winding. Thus we could use existing CT without any modification. Fig. 1 shows the measurement circuit used to verify the operation of this device.

The waveforms of the excitation current and secondary voltage are shown in Fig. 2. As our excitation source had low impedance (achieved by the proper design of the excitation transformer), the excitation voltage was practically undistorted sinewave. The current waveform contains odd harmonics, and in case that the DC current component is present, the core is magnetized non-symmetrically and even harmonics appear in the secondary current. 




Fig. 1: The measurement circuit to characterize CT current sensor: $\mathrm{I}_{1}$ is the (measured) primary $50 \mathrm{~Hz}$ current, $\mathrm{I}_{2}$ is the secondary current which is measured as a voltage drop across the nominal burden $R_{2}=0.2 \Omega$. $I_{\text {exc }}$ is the excitation current created by the power generator $G$ and transformer TR 1 . $I_{\text {inj }}$ is an (unwanted) primary current injected from the excitation. The DC component in the measured primary current $\mathrm{I}_{1 \mathrm{DC}}$ was simulated by injecting much lower current into the auxiliary winding of the CT. The DC source was decoupled from the AC circuit by choke L. For some of the measurements the measured current $\mathrm{I}_{1}$ was supplied by another transformer TR2.

This works well for saturated core as shown by distorted current sinewave in Fig. 2a. However, the effect is present even for much lower excitation level, when the harmonic distortion is not visible (Fig. 2b).


Fig. 2: Excitation current into the secondary winding (top trace, $100 \mathrm{~mA} / \mathrm{div}$ ) and corresponding secondary voltage $(50 \mathrm{~V} / \mathrm{div})$ for (a) $370 \mathrm{~Hz} / 80 \mathrm{~V}$ and (b) $370 \mathrm{~Hz} / 40 \mathrm{~V}$ excitation.

We therefore measured $2^{\text {nd }}$ harmonic component in the excitation current (using $0.2 \Omega$ sensing resistor) by SR 830 lock-in amplifier instead of using conventional voltage output.
The measured DC current was simulated by 15 turns winding and the DC current source was AC decoupled by choke. Single primary turn was used to simulate the influence of the impedance in the primary circuit.

The measured values are shown in Fig. 3 for short-circuited primary circuit and two amplitudes of the excitation voltage. The third characteristics was measured with open primary circuit. This is not a realistic condition, but the working point in real power grid will be always between the mentioned two extreme cases (short-circuit and open). We observed that the influence of the primary impedance is less than $10 \%$, which is acceptable for practical applications. Also the influence of the amplitude of the primary AC current is negligible for small current values below 50A, which is typical range of the measured currents.

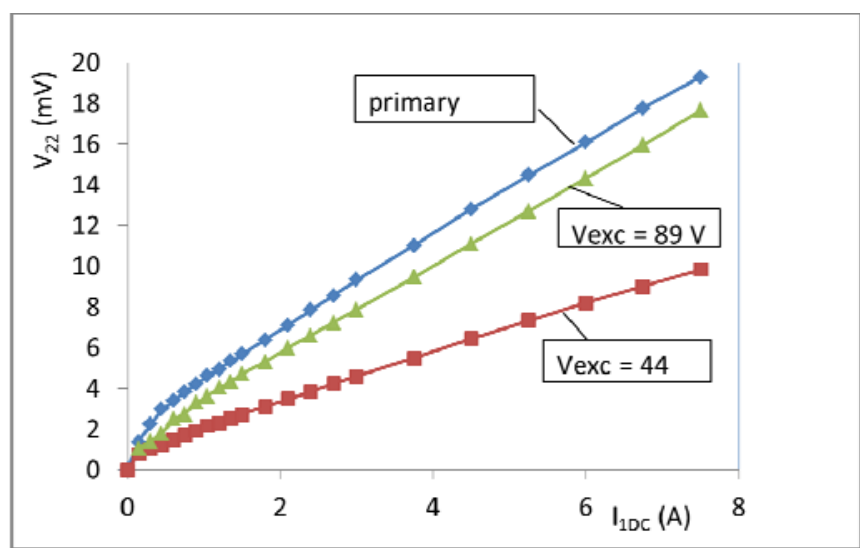

Fig. 3 Second harmonic component in the $370 \mathrm{~Hz}$ excitation current (measured as a voltage drop across $0.2 \Omega$ resistor) as a function of DC primary current. 
Fig. 4 shows the effect of increasing the excitation frequency. The primary current of $100 \mathrm{~A} / 50 \mathrm{~Hz}$ was simultaneously supplied by another transformer TR 2 with 50:1 turn ratio. The $40 \Omega$ resistor at the secondary winding of TR 2 was transformed to $40 / 50^{2} \Omega=16 \mathrm{~m} \Omega$ equivalent resistance in the primary single-turn conductor. The characteristics was measured also for the excitation frequency of $740 \mathrm{~Hz}$.

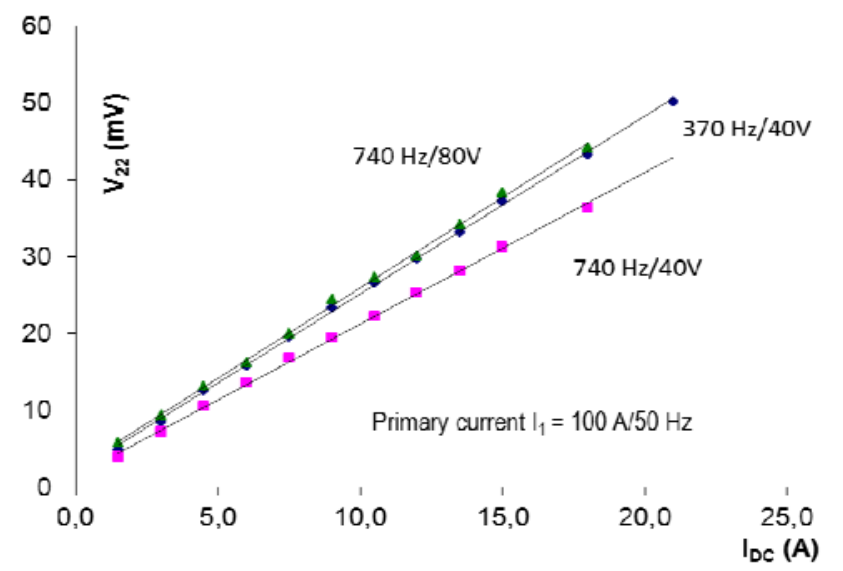

Fig. $4 \mathrm{DC}$ current response for $370 \mathrm{~Hz}$ and $740 \mathrm{~Hz}$ excitation in the presence of $50 \mathrm{~Hz} / 100$ A primary current. The primary resistance was $16 \mathrm{~m} \Omega$.

In order to achieve the same value of induction, the excitation voltage measured across the secondary winding should be double. With the same value of induction the DC sensitivity was very similar. Using higher excitation frequency has two potential advantages:

1. The $2 \mathrm{f}$ frequency can be easily filtered from $50 \mathrm{~Hz}$ component

2. The primary impedance caused by inductances of transformers in the grid is higher

We will show later that in real condition the latter benefit is probably negligible.

\section{WEAK POINTS OF THE NEW METHOD}

Our sensing method may have the following possible weak points:

A. The accuracy of the $\mathrm{CT}$ for $50 \mathrm{~Hz}$ current sensing may be deteriorated

B. The DC current sensitivity depends on the impedance in the primary current. This impedance in real power grid is hardly predictable and may vary in time

C. AC measuring current is injected into the primary circuit. The amplitude of this current also depends on the primary impedance

In the following paragraphs we will analyze the mentioned possible problems.

\section{A. Effect on $50 \mathrm{~Hz}$ CT accuracy}

First we checked two possible unwanted effects:

(i) influence of increased burden by the impedance of the excitation source and (ii) influence of the excitation current.

(i) Increasing the burden from nominal $0.2 \Omega$ to $2 \Omega$ caused aditional error below $10 \%$ even for 500 A measured current. This is acceptable, as this transformer is used for the measurement of the grid asymmetry, not for the measurement of energy. The real introduced impedance will be significantly less and mainly depends on the design of the TR1.

(ii) Aditional error of the $50 \mathrm{~Hz} \mathrm{CT}$ caused by $740 \mathrm{~Hz} / 80$ $\mathrm{V}$ excitation alone (without DC current component) was only $0.2 \%$. For DC currents below $7.5 \mathrm{~A}$ the error was below 0.5 $\% / 1^{\circ}$ for nominal (FS $=500 \mathrm{~A}$ ) AC current. This error was increasing with decreasing measured $\mathrm{AC}$ current (as usual for current transformers). The total error was below $3 \% / 1^{\circ}$ for the whole tested range of 0.05 FS to FS.

\section{B. The effect of primary impedance on injected current}

Fig. 5 shows the measured $50 \mathrm{~Hz}$ primary current with superimposed $740 \mathrm{~Hz}$ current from the excitation. The measurement was made with short-circuited primary circuit and so that the $0.7 \mathrm{~A}$ excitation current is transformed into 70 A into the primary circuit. Such a large current cannot be injected into the grid. Fortunately the situation is much better in realistic conditions.

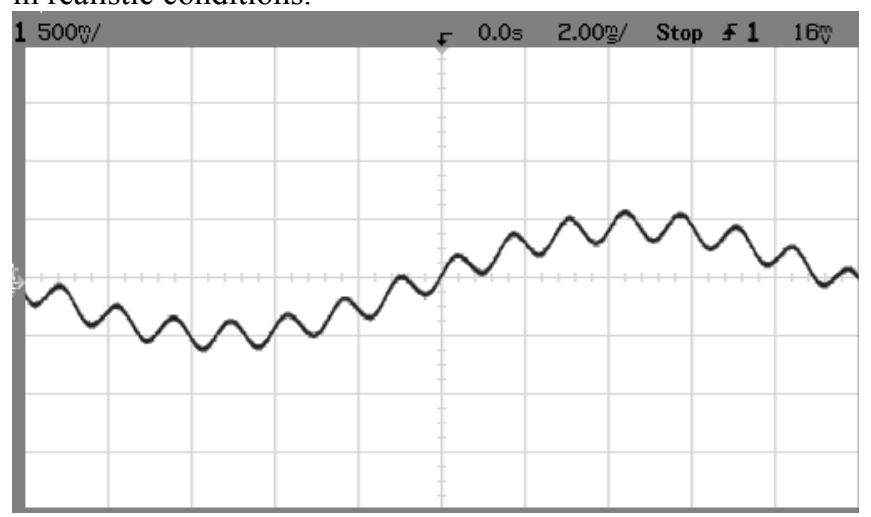

Fig. 5: $740 \mathrm{~Hz}$ injected current superimposed to the measured $50 \mathrm{~Hz}$ primary current (y scale is $500 \mathrm{~A} / \mathrm{div}$ ).

The higher the primary impedance is, the lower would be the problems with injected excitation current and DC current uncertainty.

The resistance of typical $33 \mathrm{kV}$ power grid is $0.2 \Omega /$ mile, thus one can expect that the resistive component prevail for lower frequencies. In real situations one can always expect primary resistance between $0.2 \Omega$ and $40 \Omega$ depending on the density of the grid.

Table 1 shows the value of the excitation current $I_{\text {exc }}$ and injected current $\mathrm{I}_{\text {inj }}$ as a function of excitation amplitude and frequency for four values of primary impedance $\mathrm{R}_{1}=0,0.4$, 100 and $200 \mathrm{~m} \Omega$.

TABLE I

INJECTED CURRENT FROM THE EXCITATION

\begin{tabular}{llllllll}
\hline \hline $\mathrm{f}$ & $\mathrm{V}_{2}$ & $\mathrm{R}_{1}=0 \Omega$ & \multicolumn{3}{c}{$\mathrm{R}_{1}=100 \mathrm{~m} \Omega$} & \multicolumn{2}{c}{$\mathrm{R}_{1}=200 \mathrm{~m} \Omega$} \\
$(\mathrm{Hz})$ & $(\mathrm{V})$ & $\mathrm{I}_{\text {exc }}(\mathrm{A})$ & $\mathrm{I}_{\text {inj }}(\mathrm{A})$ & $\mathrm{I}_{\text {exc }}(\mathrm{A})$ & $\mathrm{I}_{\text {inj }}(\mathrm{A})$ & $\mathrm{I}_{\text {exc }}(\mathrm{A})$ & $\mathrm{I}_{\text {inj }}(\mathrm{A})$ \\
\hline 370 & $\mathbf{4 0}$ & 0.693 & $\mathbf{7 0}$ & 0.087 & 3.96 & 0.069 & 1.98 \\
& 80 & 1.386 & 140 & 0.169 & 7.9 & 0.127 & 3.96 \\
740 & 40 & 0.355 & 36.2 & 0.086 & 3.92 & 0.068 & 1.99 \\
& $\mathbf{8 0}$ & 0.711 & $\mathbf{7 3 . 6}$ & 0.161 & 7.86 & 0.123 & 3.96 \\
1480 & 80 & 0.377 & 37.4 & 0.157 & 7.71 & 0.120 & 3.93 \\
& $\mathbf{1 6 0}$ & 0.739 & $\mathbf{7 7 . 2}$ & 0.287 & 15.4 & 0.218 & 7.9 \\
\hline \hline
\end{tabular}

Excitation current $\mathrm{I}_{\mathrm{exc}}$ and injected current $\mathrm{I}_{\mathrm{inj}}$ as a function of excitation amplitude and frequency. Bold values are corresponding to the same core induction $\mathrm{B}$ 
The impedance in the secondary circuit of the CT was the standard burden of $0.2 \Omega$ in series with the impedance of the secondary winding of the excitation transformer $\mathrm{TR}_{1}$. In order to calculate the total impedance in the equivalent circuit we should add the resistance of the secondary winding of CT, its stray inductance and resistance $R_{1}$ recalculated into the secondary by the factor of $\left(\mathrm{N}_{2} / \mathrm{N}_{1}\right)^{2}=10000$. In this case $\mathrm{R}_{1}$ is the most important from these components. For $\mathrm{R}_{1}=0$ the excitation transformer is in linear region, and $\mathrm{I}_{\mathrm{inj}} \approx \mathrm{N}_{1} / \mathrm{N}_{2} \mathrm{I}_{\mathrm{exc}}$ at all frequencies and amplitudes. This means that the transformer works as a reversed current transformer (CT). Dominant part of $\mathrm{V}_{2}$ is the induced voltage, therefore the core induction $\mathrm{B} \approx \mathrm{V}_{2} / \mathrm{f}$. Bold values in Table 1 thus correspond to the same value of $\mathrm{B}$. The excitation current required for given induction is ideally independent of frequency. In reality the current is slightly increasing (from $70 \mathrm{~mA}$ at $370 \mathrm{~Hz}$ to 77.2 at $1480 \mathrm{~Hz}$ ) which is caused by decreasing permeability. The amplitudes of both currents are linearly dependent on secondary voltage $V_{2}$ at all frequencies, indicating that stray inductances are in this case negligible. For $R_{1}=100 \mathrm{~m} \Omega$ the recalculated resistance $\mathrm{R}_{1}{ }_{1}=10000 * 0.1=1 \mathrm{k} \Omega$, and the transformer no longer behaves like CT. This is favourable situation for our purposes: the excitation current required for operation of our sensor is much lower: at $370 \mathrm{~Hz}$ the current drops by the factor of 8 . Injected current drops even more: from $70 \mathrm{~A}$ to $4 \mathrm{~A}$. In this case the transformer behaves closely to ideal voltage transformer (VT): the current is proportional to the voltage, regardless frequency. As still $\mathrm{B} \approx \mathrm{V}_{2} / \mathrm{f}$, in this mode the excitation current required to achieve given induction $\mathrm{B}$ increases with frequency.

By further increasing $R_{1}$ from $100 \mathrm{~m} \Omega$ to $200 \mathrm{~m} \Omega$ the injected current further drops down. From these measurements we can conclude, that in real situations the injected current is well below the permissible level of $2 \mathrm{~A}$.

\section{The effect of primary impedance on DC current sensitivity}

We observed that the efficient strategy to stabilize DC current sensitivity is to keep $V_{2}$ constant. The result is shown in Fig. 6: for the grid impedance range from $0.02 \Omega$ to $100 \Omega$ the sensitivity variation was below $10 \%$, which is well acceptable for our application.

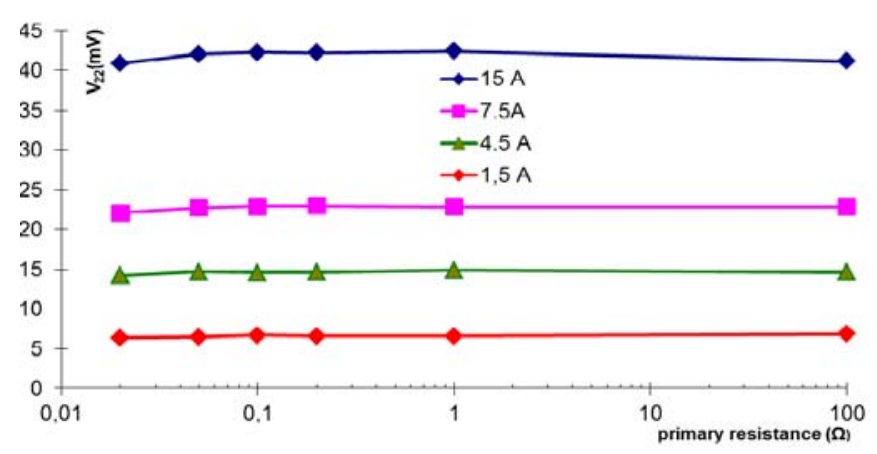

Fig. 6: Sensor response to DC current as a function of primary resistance. The excitation frequency was $370 \mathrm{~Hz}$, the excitation amplitude was always adjusted to $\mathrm{V}_{2}=80 \mathrm{~V}$.

\section{CONCLUSIONS}

We presented a method for the measurement of DC current component in the power grid using existing protection current transformers. This may be economical solution to monitor DC currents in multiple points in order to increase the grid stability. We used voltage excitation and current monitoring which allowed us to use existing single secondary winding. The advantage of our solution is that the basic operation of the current transformer for AC currents remains unaffected, with acceptable degradation of its accuracy. Any DC magnetization, which degrade the measurement accuracy [9], is demagnetized by the excitation. Achievable precision for DC current measurement is $10 \%$, which is sufficient for the monitoring and protection purposes.

The disadvantage of the described method is the circuit complexity and power consumption. The possible solution is to use pulse excitation with low duty factor, as the DC current reading should be typically upgraded only once a minute. By multiplexing both excitation and processing circuits can be used for multiple CTs in a given location. The used lock-in amplifier can be replaced by integrated solution such as $A D$ 630 .

Even though the fluxgate principle was described in 1930's, and the DC current comparator based on this principle was developed in 1960's, it is still the most precise roomtemperature method allowing evaluating DC electric current with ppm precision. Other methods for flux modulation were developed since that time such as magnetostrictive current sensors [10], but never reached industrial applications. We show that fluxgate current sensing principle can be applied with benefits also for protection purposes.

\section{REFERENCES}

[1] Campbell W.C., and Zimmerman J.E.,: Induced Electric Currents in the Alaska Oil Pipeline Measured by Gradient Fluxgate and SQUID Magnetometers, IEEE Trans. Geosci and Rem. Sen., 18 (1980), pp. 244250.

[2] S. Ziegler, R.C. Woodward, HHC Iu, et al.: Current Sensing Techniques: A Review, IEEE Sensors Journal 9 (2009) pp.354-376

[3] B. Han; T. Zhang; K. Zhang; B. Yao; X. Yue; D. Huang; H. Ren; X. Tang: Giant Magnetoimpedance Current Sensor With Array-Structure Double Probes, IEEE Trans. Magn. 44 (2008), 605-608

[4] P. Ripka, Electric current sensors: a review, Meas. Sci. Instrum. 21 (2010) pp.1-23

[5] Chi-Shan $\mathrm{Yu}$, Detection and Correction of Saturated Current Transformer Measurements Using Decaying DC Components IEEE Trans. Power Delivery 25 (2010), 1340-1346

[6] Buticchi G., Lorenzani E., Franceschini G.: A DC Offset Current Compensation Strategy in Transformerless Grid-Connected Power Converters, IEEE Trans. Pow. Delivery 26 (2011), pp 2743-2751 DOI: 10.1109/TPWRD.2011.2167160

[7] P. Ripka, P. Kaspar, P. Mlejnek: Current transformer can detect DC current component, Book of Abstracts, SMM 2011 conference

[8] G.Velasco-Quesada, A. Conesa-Roca, and F. Jeréz: Design of a LowConsumption Fluxgate Transducer for High-Current Measurement Applications, IEEE Sensors Journal 11 (2010), 280-287

[9] Koga F, Tadatsu T, Inoue J and Sasada I 2009 A new type of current sensor based on inverse magnetostriction for large current detection, IEEE Trans. Magn. 45, 4506-9

[10] Draxler K., Stybliková R.: Effect of Magnetization on Instrument Transformers Errors. Journal of Electrical Engineering Vol. 61 (2010) No $7 / \mathrm{S}$, pp. $50-53$ 